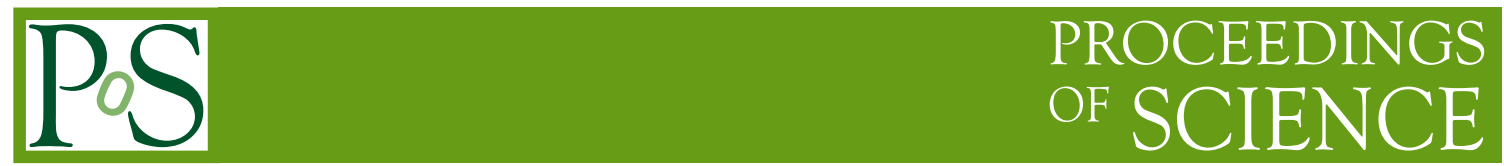

\title{
Gravitational Waves from Accreting Sources
}

\author{
Rosa Poggiani* \\ Università di Pisa and Istituto Nazionale di Fisica Nucleare, Sezione di Pisa \\ E-mail: rosa.poggiani@unipi.it
}

\begin{abstract}
Accreting systems are ubiquitous in astrophysics and are observed through the whole electromagnetic spectrum. In addition to being multifrequency astronomical sources, accreting systems are also multimessenger sources, being potential gravitational wave emitters. This paper reviews the gravitational wave emission of these sources produced by their binary nature and by other physical mechanisms, showing that it encompasses astronomical sources at all scales and over the whole gravitational spectrum.
\end{abstract}

Accretion Processes in Cosmic Sources - II - APCS2018

3-8 September 2018

Saint Petersburg, Russian Federation

${ }^{*}$ Speaker. 


\section{Introduction}

The observations of gravitational waves from binary black hole mergers and a binary neutron star merger has opened the era of gravitational and multi-messenger astronomy [3], [4], [6], [7], [8], [9], [15]. The spectrum of gravitational waves, shown in Fig. 1, spans several decades in frequency. The detections with the Advanced LIGO and Advanced Virgo detectors lie in the high frequency region, from a few $\mathrm{Hz}$ to some $\mathrm{kHz}$. In addition to the merger of stellar black holes and neutron stars, the region of ground based interferometers is expected to show the continuous radiation of pulsars and the burst signals of supernova collapse. Seismic noise limits the sensitivity of ground based interferometers below a few Hz. The sub-Hz region will be covered by the forthcoming space based laser interferometers LISA [22] and TianQin [63], that will be sensitive to resolved binaries, massive binary black hole mergers and the final stage of the merger of stellar black holes, that could trigger the operation of ground based interferometers in multifrequency gravitational wave astronomy [74]. The frequency region below $10^{-5} \mathrm{~Hz}$ includes the emission of supermassive binaries, the astrophysical stochastic background of coalescing supermassive black hole binaries, the cosmological background of gravitational waves from phase transition in the early universe [55], [56]. The emission in this region can be probed with pulsar timing techniques [65], [52], [62] [53].

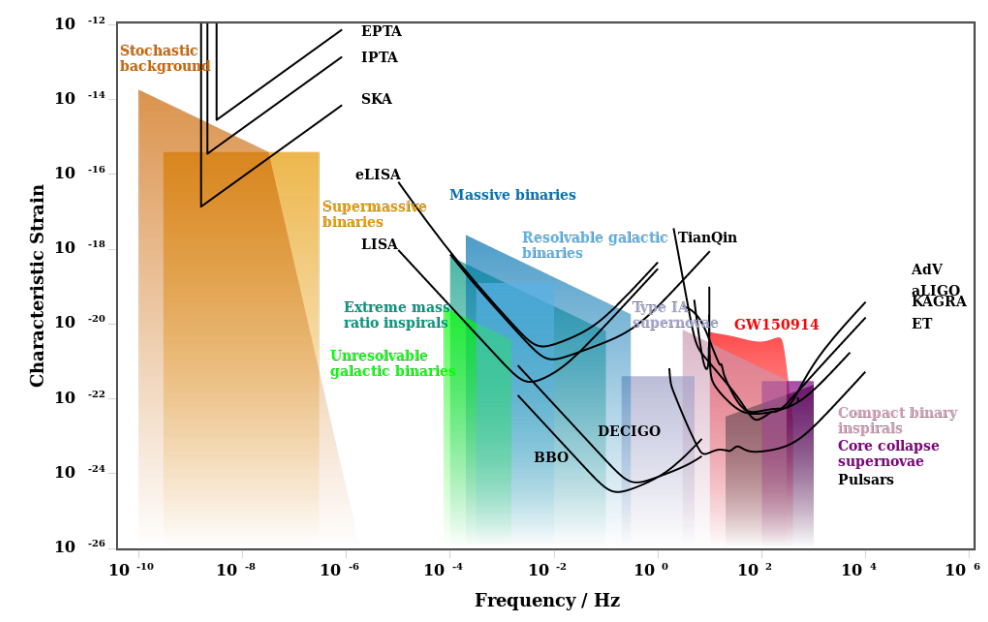

Figure 1: The spectrum of gravitational waves; credits: http://gwplotter.com/

Accretion is an ubiquitous phenomenon in astrophysics, appearing at all scales, from young stellar objects to white dwarfs to neutron stars to black holes of all sizes [47]. The standard model of accretion has been developed by [75]. Gravitational wave emission from accreting systems can be produced by the binary nature of systems and by processes related to accretion. In the following, it will be shown that the spectrum of candidate gravitational waves involves different observational techniques.

\section{Gravitational Waves from Binary Systems}

Accreting systems are binary systems. The emission of gravitational waves occurs at twice the 
orbital frequency and harmonics. For systems with negligible orbital eccentricity the contribution of harmonics is negligible and the gravitational wave characteristic amplitude is [78]:

$$
h=8.7 \times 10^{-21}\left(\frac{\mu}{M_{\odot}}\right)\left(\frac{M}{M_{\odot}}\right)^{\frac{2}{3}}\left(\frac{100 p c}{r}\right)\left(\frac{f}{10^{-3} H z}\right)^{\frac{2}{3}}
$$

where $M=M_{1}+M_{2}, \mu=\frac{M_{1} M_{2}}{M_{1}+M_{2}}, M_{1}, M_{2}$ are the masses of the primary and secondary components, $r$ the distance of the system and $f$ the gravitational wave frequency.

\section{Cataclysmic Variables}

Cataclysmic variables are binaries containing a white dwarf and a secondary star. The gravitational wave emission of cataclysmic variables has been discussed by [26] and [64], with samples of about 160 objects each, and by [70], for a sample of about 500 systems. The catalogue by Ritter and Kolb [72], [73] ${ }^{1}$, version 7.23, contains more than 1400 systems. While the orbital period is known for the majority of the cataclysmic variable, the primary and secondary mass are known only for about $10 \%$ of cataclysmic variables. The estimation of the missing masses, based on the approach by [77], [64] is discussed by [70]. The distances used by [70] are a compilation of distances estimated using the parallax, the nova expansion parallax and the Period-Luminosity-Colour relation [18], [19].

The gravitational strain estimated by [70] for an observation time of 2 years is reported in Fig. 2. The solid curve is the sky averaged sensitivity of the LISA design in [24], with an arm length of $2.5 \times 10^{6} \mathrm{~km}$, an acceleration noise extrapolated from LISA Pathfinder performances, binary confusion noise estimated by [22]. The dotted curve is the sensitivity of the original LISA design, with $5 \times 10^{6} \mathrm{~km}$ arm length [60] ${ }^{2}$. The dashed curve is confusion noise, an astrophysical background from unresolved binary systems [51], [30]. The most promising sources are AM CVn systems, with very short periods.

\section{Accreting Neutron Stars}

The possibility of gravitational emission from accreting neutron stars has been discussed by [86]. Accreting neutron star systems with weak magnetic fields that acquire angular momentum can become unstable. When the instability growth time is balanced by the viscous damping time, the gravitational strain is:

$$
h=3 \times 10^{-27}\left(\frac{1 \mathrm{kHz}}{\mathrm{mf}}\right)^{1 / 2}\left(\frac{F_{x}}{10^{-8} \operatorname{erg~s}^{-1} \mathrm{~cm}^{-2}}\right)
$$

where $m$ is the mode number, $F_{x}$ the time averaged $\mathrm{X}$-ray flux, $f$ the frequency of the emitted gravitational wave, that is monochromatic. The accreting neutron star should become non axially distorted, modulating the X-ray emission at the gravitational wave frequency, which is predicted to be of the order of some hundreds $\mathrm{Hz}$ [86]. The observation of the clustering of the neutron star

\footnotetext{
${ }^{1}$ http://wwwmpa.mpa-garching.mpg.de/RKcat/

${ }^{2} \mathrm{http}: / /$ www.srl.caltech.edu/šhane/sensitivity/
} 


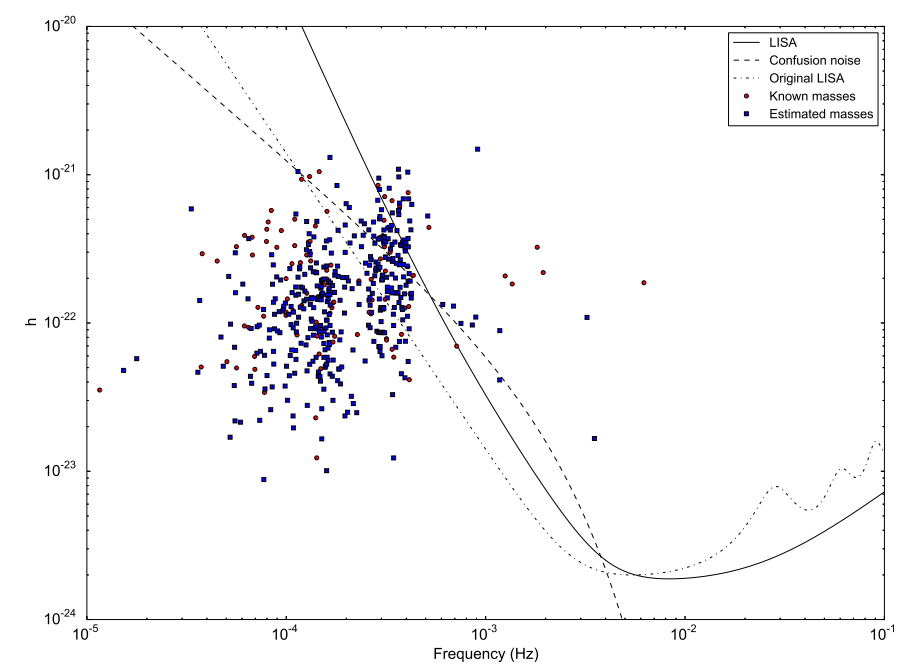

Figure 2: Gravitational wave emission of cataclysmic variables with known masses (red circles) and with estimated masses (blue squares), adapted from [70]; the solid curve is the instrumental sensitivity of the new design LISA interferometer [24], the dotted line of the original LISA [60], the dashed line is the binary confusion noise [60]

spin frequencies around $300 \mathrm{~Hz}$ suggested that the angular momentum from the accretion was converted into gravitational waves [31]. The estimated strain of accreting neutron stars is of the order of $10^{-26}$. The deformation of the crust elastic deformation due to density perturbations caused by the electron capture reactions has been addressed by [80]. The estimated quadrupole moment needed to balance the torque by accretion, is of the order of $10^{37}-10^{38} \mathrm{~g} \mathrm{~cm}^{2}$ for typical accretion rates of $10^{-10}-10^{8} \mathrm{M}_{\odot} \mathrm{yr}^{-1}$. The conditions for a steady gravitational wave emission have been discussed by [87]. Stable equilibrium will occur if the superfluid transition temperature of hyperons is below $\sim 2 \times 10^{9} \mathrm{~K}$. Gravitational radiation can drive an instability in the r-modes of young rotating neutron stars [68], leading to angular momentum loss and a decrease in the spin frequency. An instability in rotating neutron stars, driven by gravitational radiation reaction and affecting the r-modes, sets an upper limit on the spin frequency of young neutron stars [23]. It has been estimated that a rapidly rotating neutron star can convert about $0.01 \mathrm{M}_{\odot}$ into gravitational radiation. Magnetically confined mountains on accreting neutron stars could also produce detectable gravitational waves [85], [50]. The investigation by [89] suggested that only a few systems accreting at about the Eddington rate can be detected by advanced interferometers. A larger population could be detected if the spin and orbital parameters are known in advance, since the data analysis methods are based on the folding of long data records [89]. A detailed investigation of the evolution of the low-mass X ray binary in connection with the radiation of gravitational waves has been presented by [32].

The common envelope stage of accreting neutron stars is another physical situation where gravitational wave emission can occur. Neutron stars accrete at a fraction of the Bondi-HoyleLyttleton rate, but they could be able to accrete at hypercritical rates, producing strains exceeding those of Sco X-1 [54].

According to the catalogue by [61], spin and orbital periods are known for less than $15 \%$ and 
less than $50 \%$ of systems, respectively. Precision ephemerides for Low Mass X-Ray Binaries can be estimated using optical spectroscopy and have been recently determined for Sco X-1 [45], [88] and Cyg X-2 [71].

The large amount of data in the archives of X-ray observatories allows systematic searches for periodicities, that include orbital and spin periods, but also superorbital periods and outburst intervals. Systematic search for periodicities of X-ray binaries in the X-rays have been performed by [90] (all sources of RXTE ASM archive). Targeted search for periodicities of X-ray binaries have been performed by [38], [39] (Swift BAT archive), [57] (RXTE ASM) and by [69] (Swift BAT and RXTE ASM archives).

Sco X-1 has attracted interest as a potential bright source of continuous gravitational emission. The first upper limits have been set by initial LIGO using semicoherent techniques [1]. During the runs $\mathrm{O} 1$ and of the Advanced LIGO detectors limits have been set using model based crosscorrelation search [10] and Hidden Markov Models [11], [14]. Directional unmodeled searches for persistent gravitational waves from Sco X-1 have been performed by the initial LIGO [2] and the O1, O2 runs [12], [13]. The best upper limits on Sco X-1 emission estimated by the above searches are of the order of a few units times $10^{-25}$.

\section{Neutron Star-Black Hole Systems}

So far, no Neutron Star-Black Hole merger has been detected. The upper limit on the estimated merger rate estimated with Advanced LIGO is $3600 \mathrm{Gpc}^{-3} \mathrm{yr}^{-1}$ [5]. A few candidate progenitors of merging NS/BH system have been proposed [29]: Cyg X-1, Cyg X-3, MWC 656, P13 in NGC 7793. The above systems host black hole with masses ranging from 5 to $15 \mathrm{M}_{\odot}$ and secondary stars with masses in the range from 7 to $20 \mathrm{M}_{\odot}$ [29]. Different merger rates have been estimated in the investigations of the above systems: 028-0.4 $\mathrm{yr}^{-1}$ for Cyg X-1 [27], 0.09-0.15 $\mathrm{yr}^{-1}$ for Cyg $\mathrm{X}-3$ [28], 0-0.187 $\mathrm{yr}^{-1}$ for MWC 656 [49]. MWC 656 is a peculiar source, being the first detected Be/Black hole system [34], [67], [42], [20].

\section{Galactic Center}

There is a strong evidence that a supermassive black hole with a mass of about $4 \times 10^{6} \mathrm{M}^{\odot}$, Sgr $\mathrm{A}^{*}$, is at the center of our Galaxy [43]. The possibility of persistent gravitational waves from the Galactic center have been investigated by the initial [2] and advanced LIGO interferometers [12], [13]. The best upper limits estimated by the above searches are of the order of a few units times $10^{-25}$. The observational evidence for $\mathrm{Sgr} \mathrm{A}^{*}$ is given by the orbits of the S-stars, that are examples of Extreme Mass Ratio Inspiral (EMRI) events. Being not compact, the S-stars undergo tidal disruption, but their gravitational emission during the inspiral stage can be detected with Pulsar Timing techniques [33]. The properties of $37 \mathrm{~S}$-stars orbiting Sgr A* have been presented by [46]. The work by [33] estimates the gravitational waveforms, with frequencies of the order of nanoHz and amplitudes in the range $10^{-20}-10^{-19}$, about five orders of magnitude below the sensitivity of current and planned Pulsar Timing Arrays.

Other systems emitting gravitational waves could by detected by the space based interferometer LISA. A discussion of the gravitational emission from general objects orbiting Sgr A* in 
circular orbits has been presented by [48]. The authors showed that compact objects with masses larger than $10^{-4} \mathrm{M}_{\odot}$, main sequence stars with mass lower than $2.5 \mathrm{M}_{\odot}$ can be detected by the LISA interferometer with one year observation. Inspiralling stellar mass black holes around Sgr $\mathrm{A}^{*}$ with masses of about $40 \mathrm{M}_{\odot}$ could be detected by LISA [44].

\section{Active Galactic Nuclei}

Blazars are active galactic nuclei powered by accretion onto one or a pair of supermassive black holes, that show variability over the whole electromagnetic spectrum [79], with scales ranging from minutes to decades. Blazars power radio jets that often show an apparent superluminal motion. The presence of pair of black holes, of jets and variability at small scale are all candidate sources of gravitational wave emission.

Gravitational emission can be produced by the ejection of jet superluminal components and precession of accretion disks [66], [21]. The superluminal emission in blazars and other objects (microquasars, T Tauri stars) could be produced by the superposition of the accretion disk precession induced by a central Kerr black hole and the fragmentation of the tilted disk, dynamically driven by the Bardeen-Petterson effect [25]. Gravitational wave bursts occur during the initial acceleration of the jet, with a frequency spectrum in the region of the space based interferometer LISA [66], [21].

Blazar flares can last for months, but often variability at scales of the order of minutes occurs. An example is the gamma variability within 10 minutes and with distinct bursts varying on scales of about 200 seconds observed in PKS 2155-304 by HESS in July 2006 [17]. Gravitational wave emission associated to the flare could occur at the same time of electromagnetic emission, thus could be detected by ground based interferometers [40]. The physical mechanisms of fast flares [40] could be the fragmentation of accretion disk for a blazar hosting a single supermassive black hole [59], the impact from the accretion disk of the secondary black hole in blazars hosting pairs of supermassive black holes [82], dynamical friction from satellites infalling onto a coplanar accretion disk [35].

Supermassive black hole binaries in active galactic nuclei can emit gravitational waves as binaries. The presence of a black hole pair can be inferred from periodicities in the light curve of the active galactic nucleus (see e.g. [37]). The light curve of OJ 287 is shown in Fig. 3. The photometric data record extends for more than one century, showing two peaked outbursts with a recurrence time of about 12 years that are associated to the orbital motion of the black holes. OJ 287 has been modeled as a binary black hole system with precession [76], [58], [81], [82], [83]. The timing the 2007 outburst demonstrated the energy loss via gravitational radiation [84], that include reaction effects and can provide a test for the no-hair theorem [41].

Since the periodicities of blazars are in the range from years to decades, the spectrum of the gravitational radiation contains frequencies in the range $10^{-9}-10^{-8} \mathrm{~Hz}$, the domain of pulsar timing.Limits on the gravitational wave emission from supemassive black hole binaries [16] and OJ 287 [36] have been set. 


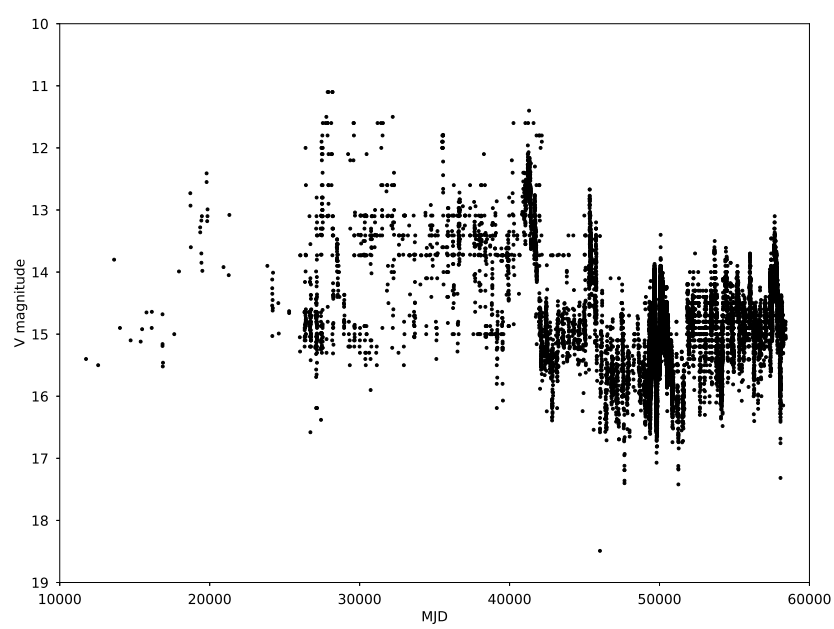

Figure 3: V band light curve of OJ 287 (data from http://altamira.asu.cas.cz/iblwg/data/oj287/ and AAVSO)

\section{Conclusions}

Accreting systems are potential gravitational wave emitters, covering the domain of cataclysmic variables, X-ray binaries, active galactic nuclei. The emission occurs in different freqeuncy regions, demanding for different observational techniques: ground based interferometry, space based interferometry pulsar timing.

\section{References}

[1] J. Aasi et al., PRD 91 (2015) 062008.

[2] J. Abadie et al, PRL 107 (2011) 271102.

[3] B. P. Abbott et al., PRL 116 (2016) 061102.

[4] B. P. Abbott et al., PRL 116 (2016) 241103.

[5] B. P. Abbott et al., ApJL 832 (2016) L21,

[6] B. P. Abbott et al., PRL 118 (2017) 221101.

[7] B. P. Abbott et al., ApJ 851 (2017) L35.

[8] B. P. Abbott et al., PRL 119 (2017) 141101.

[9] B. P. Abbott et al., PRL 119 (2017) 161101.

[10] B. P. Abbott et al., ApJ 847 (2017) 47.

[11] B. P. Abbott et al., PRD 95 (2017) 122003.

[12] B. P. Abbott et al., PRL 118 (2017) 121102.

[13] B. P. Abbott et al., $P R D 100$ (2019) 062001.

[14] B. P. Abbott et al., PRD 100 (2019) 122002. 
[15] B. P. Abbott et al., PRX 9 (2019) 031040.

[16] K. Aggarwal et al., ApJ 880 (2019) 116.

[17] F. Aharonian et al., ApJ 644 (2007) L71.

[18] T. Ak et al., NewA 12 (2007) 446.

[19] T. Ak et al., NewA 13 (2008) 133.

[20] B. Aleksic et al., A\&A 576 (2015) 36.

[21] W. D. Alfonso et al., AN 336 (2015) 815.

[22] P. Amaro-Seoane et al., arXiv:1702.00786.

[23] N. Andersson et al., ApJ 510 (1999) 846.

[24] S. Babak et al., PRD 95 (2017) 103012.

[25] J. M. Bardeen and J. A. Petterson, ApJ 195 (1975) L65.

[26] F. Barone et al., GRG 24 (1992) 323.

[27] K. Belczynski et al., ApJ 742 (2011) L2.

[28] K. Belczynski et al., ApJ 764 (2013) 96.

[29] B. Beldycki and K. Belczynski, AcA 66 (2016) 347.

[30] P. L. Bender and D. Hils, CQG 14 (1997) 1439.

[31] L. Bildsten, ApJ 501 (1998) L89.

[32] G. S. Bisnovatyi-Kogan, Astroph 32 (1990) 176.

[33] B.-G. Cai et al., Comm. Theor. Phys. 70 (2018) 735.

[34] J. Casares et al., Nat 505 (2014) 378.

[35] P. Chang et al., ApJ 684 (2008) 236.

[36] J.-W. Chen and Y. Zhang, MNRAS 481 (2018) 2249.

[37] S. Ciprini et al., in Volume 288 - Accretion Processes in Cosmic Sources (APCS2016) - ACCRETION ONTO WHITE DWARFS, NEUTRON STARS \& BLACK HOLES, PoS(APCS2016)044.

[38] R. Corbet et al., PThPS 169 (2007) 200.

[39] R. H. D. Corbet and H. A. Krimm, ApJ 778 (2013) 45.

[40] S. Desai et al., CQG 25 (2008) 184024.

[41] L. Dey et al., ApJ 866 (2018) 11.

[42] S. A. Dzib et al., $A \& A \mathbf{5 8 0}$ (2015) 6.

[43] A. Eckart et al., Found. Phys. 47 (2017) 553.

[44] R. Emami and A. Loeb, arXiv:1903.02579v3.

[45] D. K. Galloway et al., ApJ 781 (2014) 14.

[46] S. Gillessen et al., ApJ 837 (2017) 30. 
[47] F. Giovannelli, in Volume 342 - Accretion Processes in Cosmic Sources - II (APCS2018), PoS(GOLDEN 2017)001.

[48] E. Gourgoulhon et al., $A \& A 627$ (2019) A92.

[49] M. Grudzinska et al., MNRAS 452 (2015) 2773.

[50] B. Haskell et al., MNRAS 450 (2015) 2302.

[51] D. Hils et al., ApJ 360 (1990) 65.

[52] G. Hobbs et al., CQG 27 (2010) 084013.

[53] G. Hobbs and S. Dai, NSR 4 (2017) 707.

[54] A. M. Holgado et al., ApJ 857 (2018) 38.

[55] F. Jenet et al., ApJ 625 (2005) L123.

[56] F. Jenet et al., ApJ $\mathbf{6 5 3}$ (2006) 1571.

[57] M. M. Kotze and P. A. Charles, MNRAS 420 (2012) 1575.

[58] H. J. Lehto and M. J. Valtonen, ApJ 460 (1996) 207.

[59] Y. Levin et al., astro-ph/0307084.

[60] LISA Study Team, 1998, in LISA Pre-Phase A Report. 2nd Edition, Publication MPQ-233 (1998) Max-Plank Institute for Quantum Optics, Garching.

[61] Q. Z. Liu et al., $A \& A 469$ (2007) 807.

[62] A. Lommen, RPP 78 (2015) 124901.

[63] J. Luo et al., CQG 32 (2016) 035010.

[64] M. T. Meliani et al., $A \& A 358$ (2000) 417.

[65] C. J. Moore et al., CQG 32 (2015) 055004.

[66] H. J. Mosquera Cuesta et al., OAJ 4 (2011) 98.

[67] P. Murar-Adrover et al, ApJ 829 (2016) 101.

[68] B. J. Owen et al., $P R$ D 58 (1998) 084020.

[69] R. Poggiani, in Volume 233 - Swift: 10 Years of Discovery, (SWIFT 10), PoS(SWIFT 10)157.

[70] R. Poggiani, in Volume 315 - The Golden Age of Cataclysmic Variables and Related Objects IV (GOLDEN 2017), PoS(GOLDEN 2017)008.

[71] S. S. Premachandra et al., ApJ 823 (2016) 106.

[72] H. Ritter and U. Kolb, $A \& A 404$ (2003) 301.

[73] H. Ritter and U. Kolb, Acta Polytech. CTU Proc. 2 (2015) 21.

[74] A. Sesana, PRL 116 (2016) 231102.

[75] Ni. I. Shakura and R. A. Sunyaev, A\&A 24 (1973) 337.

[76] A. Sillanpää et al., ApJ 325 (1988) 628.

[77] D. A. Smith and V. S. Dhillon, MNRAS 301 (1998) 767. 
[78] K. S. Thorne, in Three Hundreds Years of Gravitation (1987) 330, Cambridge University Press, Cambridge, eds. S. Hawking and W. Israel.

[79] M. Urry and P. Padovani, PASP 107 (1995) 803.

[80] G. Ushomirsky et al., MNRAS 319 (2000) 902.

[81] M. J. Valtonen et al., ApJL 643 (2005) L9.

[82] M. J. Valtonen et al., ApJ 646 (2006) 36.

[83] M. J. Valtonen, ApJ 657 (2007) 1074.

[84] M. J. Valtonen et al., Nat 452 (2008) 851.

[85] M. Vigelius and A. Melatos, MNRAS 395 (2009) 1972.

[86] R. V. Wagoner, ApJ 278 (1984) 345.

[87] R. V. Wagoner, ApJ 578 (2002) L63.

[88] L. Wang et al., MNRAS 478 (2018) 5174.

[89] A. L. Watts et al., MNRAS 389 (2008) 839.

[90] L. Wen et al., ApJS 163 (2006) 372. 